\title{
Spin and phase coherence lengths in InAs wires with diffusive boundary scattering
}

\author{
R. L. Kallaher* and J. J. Heremans ${ }^{\dagger}$ \\ Department of Physics, Virginia Tech, Blacksburg, Virginia 24061, USA \\ W. Van Roy and G. Borghs \\ IMEC, Kapeldreef 75, B-3001, Leuven, Belgium \\ (Received 6 June 2013; revised manuscript received 20 September 2013; published 7 November 2013)
}

\begin{abstract}
Measurements of low-temperature magnetotransport in lithographic wires of submicron widths fabricated from high-mobility AlGaSb/InAs/AlGaSb two-dimensional electron system heterostructures are presented. The dependence of the spin and phase coherence lengths on wire width and diffusion constant is investigated by analyzing the conductance in low applied magnetic fields with antilocalization models. Predominantly diffusive boundary scattering is deduced from the magnitude and wire width dependence of the conductance. Diffusive boundary scattering leads to a diffusion constant decreasing with wire width and hence allows the dependence of spin coherence on wire width and diffusion constant to be investigated concurrently. The spin coherence lengths are experimentally found to be proportional to the ratio of the diffusion constant to wire width. The phase coherence lengths follow Nyquist decoherence for low-dimensional wires.
\end{abstract}

DOI: 10.1103/PhysRevB.88.205407

PACS number(s): 73.63.Nm, 72.25.Rb, 73.20.Fz, 73.23.Ad

\section{INTRODUCTION}

Indium arsenide two-dimensional electron systems (2DESs) have received substantial interest in spintronics and spin physics due to their high electron mobilities and strong Rashba spin-orbit interaction (SOI). In reduced dimensions, InAs wires and wires of other narrow-band-gap semiconductors with strong SOI are moreover being pursued as hosts for quantum states showing non-Abelian Majorana properties under proximity-induced superconductivity. ${ }^{1-4}$ Given this interest, we probe spin-related phenomena in narrow InAs wires fabricated from high-mobility InAs 2DESs. This work examines how the low-temperature spin coherence length evolves with wire width and is influenced by boundary scattering in lithographic InAs wires. We also briefly discuss the quantum phase coherence length and its dependence on wire width and diffusion coefficient in the wires.

We model magnetotransport measurements, performed at low magnetic fields applied perpendicularly to the plane of the 2DES, in antilocalization theory in order to investigate the dependence on wire width $w$ of the spin coherence length $L_{S}$ in wires of submicron widths fabricated from high-mobility InAs 2DESs. At low temperatures, interference between phase coherent electrons on time-reversed trajectories can affect a material's conductance $G .^{5-11}$ Under strong SOI, the magnitude of the interference corrections to $G$ are sensitive to both $L_{S}$ and the phase coherence length $L_{\phi}$. Antilocalization theory provides quantitative predictions for the dependence of $G$ on $L_{S}$ and $L_{\phi}$ as a function of the applied magnetic field $B .^{5-11}$ A comparison of the experimental $G(B)$ with antilocalization theory adapted to low-dimensional systems thus provides quantitative information about $L_{S}$ and $L_{\phi}$. Our wires are in the quasi-one-dimensional (Q1D) regime, where $w$ is shorter than the mobility mean-free path $l_{e}$, and comparable to or shorter than $L_{S}$ and $L_{\phi}$.

Antilocalization in InAs wires was previously studied, either in epitaxially grown nanowires ${ }^{12-14}$ or in wires fabricated lithographically from a 2DES. ${ }^{15}$ Whereas these studies focused on systems with comparatively low electron mobilities, we report measurements on wires fabricated from InAs 2DESs with a long mean-free path $l_{e}>w$ and investigate $L_{S}$ in a system where diffusive boundary scattering dominates the overall momentum scattering. Several recent investigations of spin coherence in narrow wires with strong SOI exist, either theoretical ${ }^{5,16,17}$ or experimental on III-V semiconductor systems $^{14,18-22}$ or on surface states of bismuth. ${ }^{23}$ These investigations indicate that $L_{S}$ increases as $w$ is reduced. Diffusive boundary scattering is important in narrow InAs wires due to the fact that surface states pin the Fermi energy $E_{F}$ above the conduction band at the surface of InAs. ${ }^{24-27}$ Hence, unlike in many other III-V semiconductor systems, no depletion layer forms, and the electrons fully sample the boundary roughness. The effect of boundary scattering on spin decoherence in InAs wires may be similar to the effect in narrow metal wires, where experiments demonstrate that the spin decoherence rate is increased by boundary scattering. ${ }^{28}$ The experiments following show that the absence of depletion layer, and the resulting increased diffusive boundary scattering indeed can influence spin coherence in lithographic InAs wires. In the InAs wires discussed in this paper, the spin coherence length is thus influenced by $w$ in two competing ways: by the reduced dimensionality and by the increased boundary scattering.

\section{EXPERIMENT}

Magnetotransport through sets of narrow wires fabricated from two separate $\mathrm{Al}_{x} \mathrm{Ga}_{1-x} \mathrm{Sb} / \mathrm{InAs} / \mathrm{Al}_{x} \mathrm{Ga}_{1-x} \mathrm{Sb}$ 2DES heterostructures was measured using standard four-terminal lowfrequency lock-in techniques at $T=0.4 \mathrm{~K}$. $B$ was applied perpendicularly to the plane of the 2DES, as appropriate for antilocalization measurements aimed at deducing the effect of $w$ on $L_{S}$ and $L_{\phi}$ in a 2DES. ${ }^{15,19-23}$ Both heterostructures are grown by molecular beam epitaxy on (001) GaAs substrates. Compositionally, the two heterostructures (Fig. 1) are nominally equivalent except for the percentage of $\mathrm{Al}$ in the barrier layers $x=0.3(0.2)$ for heterostructure A (B). Within 


\begin{tabular}{|c|}
\hline $\mathrm{Al}_{\mathrm{X}} \mathrm{Ga}_{1-\mathrm{x}} \mathrm{Sb}(20 \mathrm{~nm})$ \\
\hline $\mathrm{Al}_{\mathrm{x}} \mathrm{Ga}_{1-\mathrm{x}} \mathrm{Sb}(200 \mathrm{~nm})$ \\
\hline $\mathrm{GaSb} / \mathrm{AlSb}$ S.L. $(50 \mathrm{~nm})$ \\
\hline $\mathrm{GaSb}(500 \mathrm{~nm})$ \\
\hline $\mathrm{Al}_{\mathbf{0 . 2 0}} \mathrm{Ga}_{0.80} \mathrm{Sb}(1100 \mathrm{~nm})$ \\
\hline Buffer Layers \\
\hline GaAs $(001)$ \\
\hline
\end{tabular}

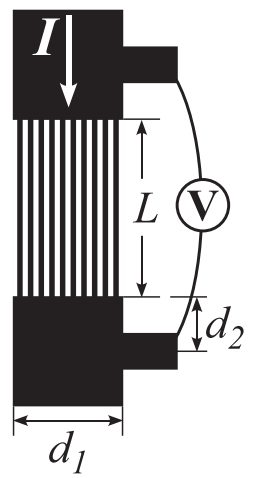

FIG. 1. Left: Schematic of the $\mathrm{Al}_{x} \mathrm{Ga}_{1-x} \mathrm{Sb} / \mathrm{InAs} / \mathrm{Al}_{x} \mathrm{Ga}_{1-x} \mathrm{Sb}$ heterostructures. The composition of the buffer layers for heterostructure $\mathrm{A}(x=0.3)$ is as follows: $1100 \mathrm{~nm} \mathrm{Al}_{0.2} \mathrm{Ga}_{0.8} \mathrm{Sb} / 32 \mathrm{~nm}$ $\mathrm{AlSb} / 5 \mathrm{~nm} \mathrm{AlAs} / 1000 \mathrm{~nm}$ GaAs; for heterostructure B $(x=0.2)$ the buffer layers lack the AlSb and AlAs layers. The GaSb/AlSb superlattice (S.L.) in both heterostructures consists of 10 periods of $5 \mathrm{~nm} \mathrm{GaSb} / 5 \mathrm{~nm} \mathrm{AlSb}$. Right: Schematic illustration of a wire set geometry. From the ratio $d_{2} / d_{1} \approx 0.5$ we estimate the total series contact resistance $R_{c} \approx \rho_{2 D}$.

$\sim 15 \%$ variability between cooldowns, the results of electrical transport measurements on the unpatterned 2DESs at $T=$ $0.4 \mathrm{~K}$ are tabulated in Table I. Due to stronger confinement, heterostructure A $(x=0.3)$ has higher electron density, but lower mobility $\mu_{2 D}$ compared to heterostructure $\mathrm{B}(x=0.2)$. Table I contains parameters calculated assuming nonparabolicity, a $\Gamma$-point electron effective mass $m^{*}=0.024 m_{e}$ (with $m_{e}$ the free-electron mass), ${ }^{29}$ and a low- $T$ energy gap of $418 \mathrm{meV}$. The values of the Fermi wavelengths $\lambda_{F}$ indicate that between 6 to 39 transverse subbands are expected to be occupied in the wires, and hence effects of transverse quantization can be neglected.

Wire sets were fabricated from each heterostructure using electron-beam lithography and reactive ion etching in a boron trichloride inductively coupled plasma. Each wire set contains 10 parallel wires of identical dimensions: the individual wires have a width $w$ and length $L=24 \mu \mathrm{m}$. The difference between sets of wires resides in $w$ with $0.17 \mu \mathrm{m} \leqslant w \leqslant 0.98 \mu \mathrm{m}$. Here, $w$ has been determined through scanning electron microscope (SEM) measurements on prototype wire sets

TABLE I. Transport and quantum well parameters of the unpatterned 2DESs in heterostructures $\mathrm{A}$ and $\mathrm{B}$, at $T=0.4 \mathrm{~K}$. Fraction $\mathrm{Al}$ in the barriers, $x$; sheet resistance, $\rho_{2 D}$; electron density, $N_{S}$; mobility, $\mu_{2 D}$; mobility mean-free path, $l_{e}$; diffusion constant, $D_{2 D}$; Fermi energy, $E_{F}$; effective mass, $m^{*}$; Fermi wavelength, $\lambda_{F}$.

\begin{tabular}{lcc}
\hline \hline & $\mathrm{A}$ & $\mathrm{B}$ \\
\hline$x$ & 0.3 & 0.2 \\
$\rho_{2 D}(\Omega / \square)$ & 42 & 26 \\
$N_{S}\left(10^{15} \mathrm{~m}^{-2}\right)$ & 9.9 & 6.7 \\
$\mu_{2 D}\left(\mathrm{~m}^{2} / \mathrm{Vs}\right)$ & 15.0 & 36.0 \\
$l_{e}(\mu \mathrm{m})$ & 6.43 & 11.2 \\
$D_{2 D}\left(\mathrm{~m}^{2} / \mathrm{s}\right)$ & 2.39 & 3.65 \\
$E_{F}(\mathrm{meV})$ & 67.9 & 47.6 \\
$m^{*} / m_{e}$ & 0.10 & 0.084 \\
$\lambda_{F}(\mathrm{~nm})$ & 25.2 & 30.6 \\
\hline \hline
\end{tabular}

fabricated in the same manner as the wires presented here. We note that, as mentioned previously, $E_{F}$ at the surface of InAs is pinned above the conduction band by surface states. ${ }^{24-27}$ Thus, carrier depletion at the edge of the wires is expected to be minimal and the conducting and lithographic widths of the wires are assumed to be equivalent.

The data are presented in terms of conductance per wire $G \equiv 1 / R$, with $R$ denoting the resistance per wire. In order to accurately determine $G$, we estimate, based on device geometry, the effective series contact resistance $R_{c}$ due to the contact areas at both ends of the wires (Fig. 1). The centers of the voltage probes are separated from the ends of the wires by a distance $d_{2} \approx 7.5 \mu \mathrm{m}$ and the corresponding contact areas have a width $d_{1} \approx 15 \mu \mathrm{m}$. Since the contact areas on both sides of the wires contribute to the total $R_{c}$, we estimate $R_{c}=2\left(d_{2} / d_{1}\right) \rho_{2 D} \approx \rho_{2 D}$, where $\rho_{2 D}$ denotes the sheet resistance of the unpatterned 2DES. From this estimate we determine $R=\left(R_{T}-R_{c}\right) / N_{p}$ where $R_{T}$ is the total measured resistance and $N_{p}=10$ is the number of parallel wires in each wire set.

According to the Einstein relation, the classically predicted conductance at $B=0$ is given by $G_{0} \equiv(w / L) \rho_{2 D}^{-1}=$ $(w / L) 2 e^{2} v_{0} D$ where $D$ denotes the wire's diffusion constant and $\nu_{0}$ is the density of states per spin orientation at $E_{F}$. For purely specular boundary scattering, $D$ is unchanged from the two-dimensional expression: $D=D_{2 D}=\frac{1}{2} v_{F} l_{e}$, where $v_{F}$ represents the Fermi velocity. Thus, specular boundary scattering would for the measured $G(B=0)=G(0)$ lead to the simple expression $G(0) \rightarrow G_{0}=(w / L) \rho_{2 D}^{-1}$ for all $w$. However, diffusive scattering from the boundaries can reduce $D$ in narrow wires. According to the boundary scattering model given in Ref. 30, the ratio $D / D_{2 D}$ for purely diffusive boundary scattering in narrow wires is expressed as ${ }^{30}$

$$
\frac{D}{D_{2 D}}=1-\frac{4 l_{e}}{\pi w} \int_{0}^{1} x \sqrt{1-x^{2}}\left[1-\exp \left(-\frac{w}{x l_{e}}\right)\right] d x
$$

According to Eq. (1), $D$ rapidly drops below $D_{2 D}$ as $w$ is reduced below $\sim 2 l_{e}$ in narrow wires with diffusive boundary scattering. The actually measured $G(0)$ as a function of $w$ is plotted for both heterostructures in Figs. 2(a) and 2(b). Using the Einstein relation along with Eq. (1), theoretical plots for purely specular and purely diffusive boundary scattering are displayed along with the measured data.

Figure 2 shows that the measured $G(0)$ are significantly less than predicted for specular boundary scattering $[G(0) \rightarrow$ $\left.G_{0}=(w / L) \rho_{2 D}^{-1}\right]$. In contrast, accurate predictions of $G(0)$ can be achieved using Eq. (1). The ratio $D / D_{2 D}$ for the different $w$ is experimentally determined from the Einstein relation and the measured values of $G(0)$ and $\rho_{2 D}\left[D / D_{2 D}=G(0) L \rho_{2 D} / w\right]$. These experimental values of $D / D_{2 D}$ are used throughout the remainder of this work and are displayed and compared with Eq. (1) in Fig. 2(c). We note that Eq. (1) does appear to underestimate $D / D_{2 D}$ for large $w / l_{e}$. The reason for the discrepancy is unknown and is outside the scope of the work presented in this paper. Regardless, since the measured $G(0)$ for all $w$ are significantly less than predicted assuming entirely specular boundary scattering, we conclude that scattering from the boundaries is, in fact, predominantly diffusive. In terms of the fits to antilocalization theory, the value of $D$ and the nature 

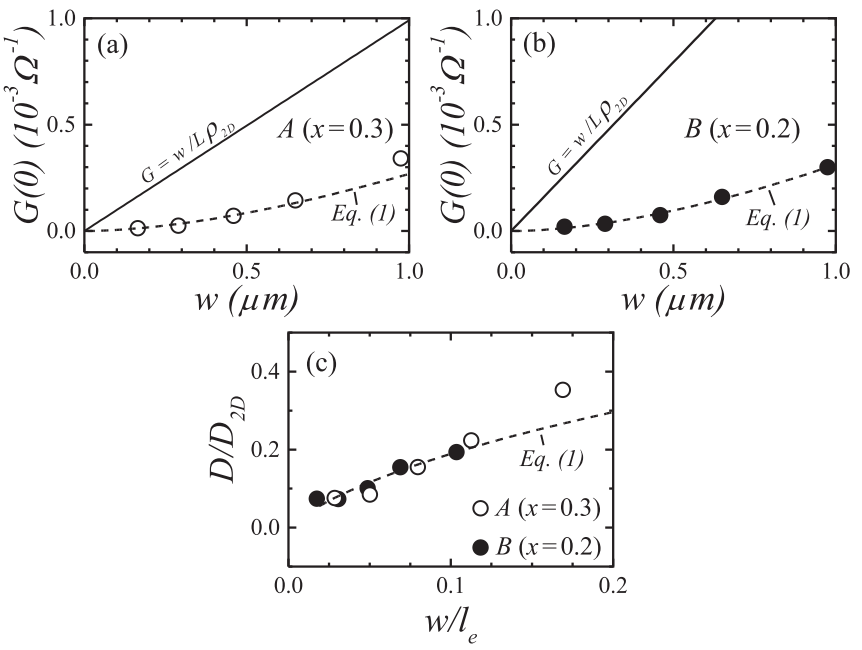

FIG. 2. Dependence on wire width $w$ of the measured conductance in zero applied magnetic field $G(0)$ for wires fabricated from (a) heterostructure A (open circle) and (b) heterostructure B (filled circle). Solid and dashed lines in (a) and (b), respectively, depict the predicted $w$ dependence for purely specular boundary scattering $\left(G_{0}=w / L \rho_{2 D}\right)$ and for purely diffusive boundary scattering [derived from Eq. (1)]. (c) Values for the diffusion constant $D / D_{2 D}$ deduced from the measured $G(0)$ data displayed in (a) and (b). The data show good agreement with Eq. (1).

of the boundary scattering is important when calculating the effective value of the magnetic length $L_{B}$. In addition, as will be shown, the $w$ dependence of $D$ is found to directly impact the $w$ dependencies of $L_{S}$ and $L_{\phi}$.

The magnetoconductance $\Delta G \equiv G(B)-G(0)$ obtained at $T=0.4 \mathrm{~K}$ for wires fabricated from the two InAs 2DESs are depicted in Fig. 3. Except for the narrowest wires fabricated from heterostructure $\mathrm{A}(w=0.17 \mu \mathrm{m})$, wires fabricated from both heterostructures display the characteristic shape of antilocalization: $\Delta G$ first decreases as $|B|$ is initially increased from zero until $\Delta G$ reaches a minimum at the field $B_{\text {min }}$, directly above which $\Delta G$ increases as $|B|$ increases further. The data for the narrowest wires fabricated from heterostructure $\mathrm{A}$ ( $w=$ $0.17 \mu \mathrm{m}$ ) will not be further analyzed since antilocalization is suppressed and not observed in these wires.

\section{ANALYSIS AND RESULTS}

At $B=0$ and in the absence of SOI, the quantum correction per spin channel to the two-dimensional conductivity due to time-reversed trajectories in a Q1D system can be expressed as $^{31,32}$

$$
\delta\left(\rho_{2 D}^{-1}\right)=-\frac{e^{2}}{h} \frac{L_{\phi}}{w} .
$$

An applied $B$ introduces time-reversal-symmetry breaking, expressed by a modification of $L_{\phi}$ to include a magnetic length appropriate for the particular Q1D system $L_{B} \equiv \sqrt{D \tau_{B}}$, where $\tau_{B}$ represents a magnetic field dephasing time..$^{5,11,30,33,34}$ Under SOI, the role of $L_{\phi}$ is assumed by a combination of effective singlet and triplet ${ }^{5,7,10,11,22,35}$ length scales. The resulting singlet and triplet length scales $L_{s, m}$ (with $m= \pm 1,0$

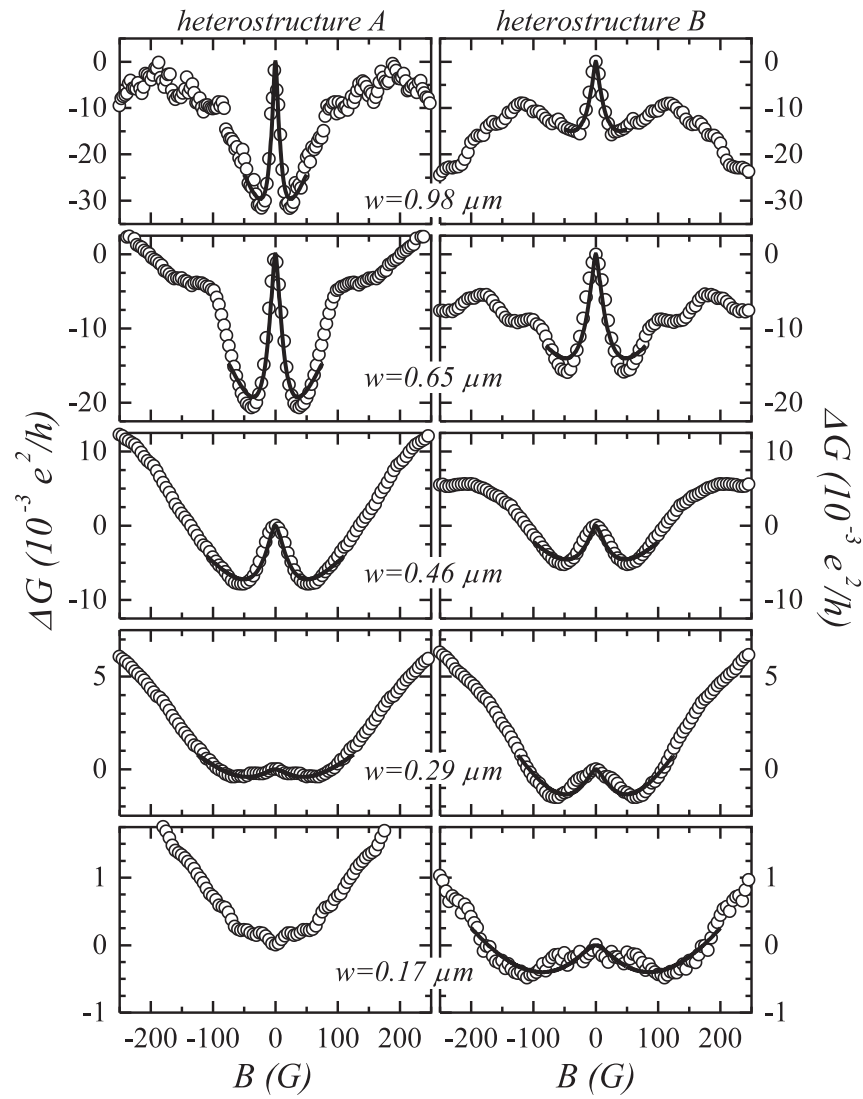

FIG. 3. Measured change in conductance $\Delta G \equiv G(B)-G(0)$, at $T=0.4 \mathrm{~K}$ as function of applied magnetic field $B$ for wires of different widths ( $w$ as indicated) on heterostructure A (left column) and heterostructure B (right column). Solid lines are fits of the magnetoconductance traces to antilocalization theory.

and $s=0,1)$ are expressed as

$$
L_{s, m}\left(L_{B}\right)=\left(L_{\phi}^{-2}+v_{s, m} L_{S}^{-2}+L_{B}^{-2}\right)^{-\frac{1}{2}},
$$

where $v_{s, m}$ are constants that we take as $v_{1, \pm 1}=1, v_{1,0}=2$, and $v_{0,0}=0$, consistent with the anisotropic spin decoherence in (001) III-V 2DESs. ${ }^{8,36}$ Here, $L_{S} \equiv \sqrt{D \tau_{S}}$ with $\tau_{S}$ representing the spin decoherence time, and $L_{\phi} \equiv \sqrt{D \tau_{\phi}}$ with $\tau_{\phi}$ representing the phase decoherence time. Unlike the triplet contributions $\left(L_{1, m}\right)$, the singlet $\left(L_{0,0}\right)$ contribution is not sensitive to spin decoherence under SOI $\left(v_{0,0}=0\right) .^{10,11,22,35}$ Noting that $\delta G=(w / L) \rho_{2 D}^{-1}$, the $B$ dependence of $\delta G(B)=G(B)-G_{0}$ in Q1D systems under SOI can now be described using a combination of length scale ratios $L_{s, m} / L$ (Refs. 22 and 34):

$$
\delta G(B)=-\frac{e^{2}}{h} \frac{1}{L}\left(\sum_{m= \pm 1,0} L_{1, m}\left(L_{B}\right)-L_{0,0}\left(L_{B}\right)\right) .
$$

We note that $\Delta G=G(B)-G(0)=\delta G(B)-\delta G(0)$, and hence the experimental data can be compared to fits to Eq. (4). For simplicity, we have neglected the short-time cutoff correction for ballistic wires suggested by Beenakker. ${ }^{30}$ Including a short-time cutoff when analyzing the data leads to extracted values of $L_{S}$ that are somewhat $(\approx 20 \%)$ larger than the results presented here, but the short-time cutoff correction does not quantitatively or qualitatively impact the observed dependence of $L_{S}$ on $w$ and $D$. 

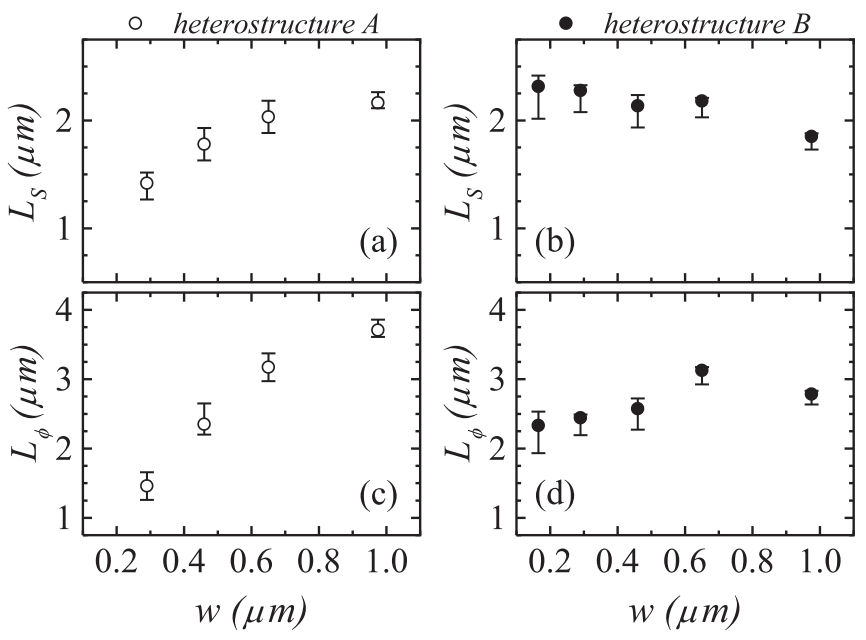

FIG. 4. Extracted values of $L_{S}$ and $L_{\phi}$ obtained from fitting the measured $\Delta G$ to the Q1D antilocalization model. (a) and (c) display the dependence of $L_{S}$ and $L_{\phi}$ for wires on heterostructure A. (b) and (d) display the dependence of $L_{S}$ and $L_{\phi}$ for wires on heterostructure $\mathrm{B}$.

For Q1D wires where $w \approx l_{m} \equiv \sqrt{\hbar / e B}$ and where $l_{e}>w$, phenomena due to modifications of the wave-function boundary conditions and ballistic flux cancellation have to be considered, and $L_{B}$ is effectively described by $22,30,33,37$

$$
L_{B}^{1 D}=l_{m} \sqrt{\left(\frac{C_{1} l_{m}^{2} l_{e}}{w^{3}}+\frac{C_{2} l_{e}^{2}}{w^{2}}\right) \frac{D}{D_{2 D}}} .
$$

The values of the numerical constants $C_{1}$ and $C_{2}$ depend on whether the boundary scattering is specular or diffusive, with $C_{1}=2 \pi$ and $C_{2}=1.5$ for predominantly diffusive boundary scattering as considered here. ${ }^{30}$ The first fraction in Eq. (5) dominates at weak applied $B\left(l_{m}>\sqrt{w l_{e}}\right)$, while the second fraction dominates at higher $B\left(l_{m}<\sqrt{w l_{e}}\right) .{ }^{30}$

In order to find values of $L_{S}$ and $L_{\phi}$ in the Q1D model, we fit the measured $\Delta G$ to Eq. (4) using Eqs. (3) and (5) to evaluate $L_{s, m}$ and $L_{B}$. The extracted values for $L_{S}$ and $L_{\phi}$ are presented in Fig. 4. The data displayed in Fig. 4 correspond to fits taken over the range $|B| \lesssim 2 B_{\min }$ and the error bars correspond to the different values of $L_{S}$ and $L_{\phi}$ obtained by fitting over different ranges of $B$ from $1.5 B_{\min }$ to $4 B_{\min }$. Results from the Q1D model indicate differing dependencies of $L_{S}$ on $w$ between wires fabricated from heterostructures A and B. For heterostructure A, $L_{S}$ decreases moderately as $w$ narrows; for heterostructure $\mathrm{B}, L_{S}$ increases moderately as $w$ narrows. As a later discussion of $L_{S}$ will clarify, the dependence of $L_{S}$ on $w$ is affected by the variation of $D$ with $w / l_{e}$ under diffusive boundary scattering, and $l_{e}$ differs for heterostructures $\mathrm{A}$ and B. The extracted $L_{\phi}$ for both heterostructures are found to decrease as $w$ narrows.

We note that the functional dependence of $\delta G(B)$ on $B$ is determined, in part, by the effective dimensionality of the system. $L_{s, m}$ decreases as $|B|$ increases and a dimensional crossover from $1 \mathrm{D}\left(w<L_{s, m}\right)$ to $2 \mathrm{D}\left(w>L_{s, m}\right)$ can occur for $L_{s, m} \approx w$ in the diffusive regime $\left(l_{e}<w\right) .{ }^{15}$ While in the narrower wires the condition $w<L_{s, m}$ is fulfilled, in our widest wires $(w=0.98 \mu \mathrm{m})$ and at the higher $B$ considered, the $L_{s, m}$ approach $w$. Yet, application of Eq. (5) experimentally provides good fits to our data. We are moreover not aware of a theoretical description of antilocalization with dimensional crossover for wires where $w<l_{e}$. We hence have analyzed $\Delta G$ without dimensional crossover in this work. The following discussion then focuses on $L_{S}$ and $L_{\phi}$ obtained by analyzing the data with the Q1D model.

\section{DISCUSSION}

In order to explain the observed dependence of $L_{S}$ on $w$ (Fig. 4), we examine the dependence of $L_{S}$ on $D$. Figures 5(a) and 5(b) display the experimentally observed dependence of $w L_{S}$ on $D$ for the wires fabricated from heterostructures A and $\mathrm{B}$, respectively, showing that to a good approximation $w L_{S}$ scales linearly with $D$, implying $L_{S} \propto D / w$. The experimental dependence of $D$ on $w / l_{e}$ has already been discussed in a previous section, and is shown in Fig. 2(c) to agree with Eq. (1), valid for diffusive boundary scattering. The differing dependencies of $L_{S}$ on $w$ in Fig. 4 follow from $L_{S} \propto D / w$, taking into account the dependence of $D$ on $w / l_{e}$. With a lower $l_{e}=6.43 \mu \mathrm{m}$ in heterostructure A, compared to $l_{e}=11.2 \mu \mathrm{m}$ in heterostructure $\mathrm{B}$, in heterostructure $\mathrm{A} w / l_{e}$ spans a wider range and $D$ has a stronger effect on $L_{S}$. In heterostructure A, the decrease in $D$ with narrowing $w$ leads to a decrease in $L_{S}$, reversing the usual increase in $L_{S}$ with narrowing $w{ }^{18-23}$ We next explore the origin of our observation that $L_{S} \propto D / w$.

We are not aware of explicit theoretical predictions of the dependence of $L_{S}$ on $D$ and $w$ in ballistic wires $\left(l_{e}>w\right)$ with diffusive boundary scattering. Yet, for systems with dominant Rashba $\mathrm{SOI}^{38}$ as will be assumed here, the $w$
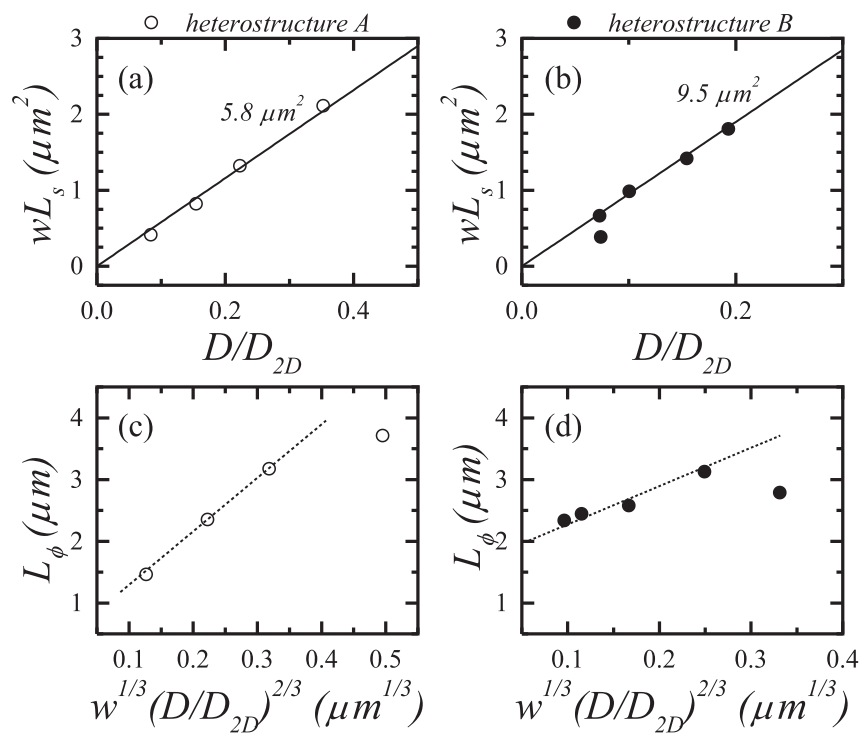

FIG. 5. Plot of $w L_{S}$ versus the diffusion constant $D$ in narrow wires fabricated from (a) heterostructure A and (b) heterostructure B. A linear dependence of $w L_{S}$ on $D$ is found in both heterostructures; the numerical values indicate the proportionality constant between $w L_{S}$ and $D / D_{2 D}$ for each heterostructure. The dependence of the phase coherence length $L_{\phi}$ on $D$ and $w$ is shown in (c) for heterostructure A and in (d) for heterostructure B. A linear dependence of $L_{\phi}$ on $D^{2 / 3} w^{1 / 3}$ is consistent with the Nyquist decoherence mechanism in Q1D systems. 
dependence of $L_{S}$ in diffusive wires (with $l_{e}<w, D=D_{2 D}$ ) under the D'yakanov-Perel (DP) spin decoherence mechanism is theoretically predicted ${ }^{5,21}$ to follow $w L_{S}=\sqrt{12} L_{\Omega}^{2}$, where the spin precession length $L_{\Omega}=v_{F} / \Omega$ with $\Omega$ representing the spin precession frequency under SOI. For systems with Rashba SOI, where the zero- $B$ spin-splitting energy is $\Delta_{\mathrm{SO}}=2 k_{F} \alpha$ (with $k_{F}=\sqrt{2 \pi N_{S}}$ denoting the Fermi wave vector and $\alpha$ the Rashba SOI parameter), we have $\hbar \Omega=2 k_{F} \alpha$ and $L_{\Omega}=$ $\hbar^{2} /\left(2 m^{*} \alpha\right){ }^{5,21,39}$ Under the DP mechanism, the spin decoherence rate of the parent $2 \mathrm{DES}$ is given by $1 / \tau_{S}^{2 D}=\Omega^{2} \tau_{e} / 2$, with $\tau_{e}$ the momentum scattering time $\left(l_{e}=v_{F} \tau_{e}\right){ }^{5,8,9,18,39}$ Then, $D \tau_{S}^{2 D}=\left(v_{F} / \Omega\right)^{2}=L_{\Omega}^{2}$. Combining $w L_{S}=\sqrt{12} L_{\Omega}^{2}$ and $D \tau_{S}^{2 D}=L_{\Omega}^{2}$, we obtain $L_{S}=\sqrt{12} D \tau_{S}^{2 D} / w$. Hence, the dependence $L_{S} \propto D / w$ is, in fact, predicted for spin decoherence due to the DP mechanism in narrow wires in the diffusive regime under Rashba SOI.

The dependence $w L_{S}=\sqrt{12} L_{\Omega}^{2}$ for wires in the diffusive regime $\left(l_{e}<w\right)$ is analogous to expressions for the magnetic length in such wires, where $w L_{B}=\sqrt{3} l_{m}^{2} \cdot{ }^{15,19,30,34}$ The analogy relates to the concept that linear SOI effects can be ascribed to an effective magnetic vector potential. Then, the dependence of $L_{S}$ on $w$ in narrow wires is expected to be similar to the dependence of $L_{B}^{1 D}$ on $w$, with $L_{\Omega}$ assuming the role of $l_{m}{ }^{5,19}$ For ballistic wires $\left(l_{e}>w\right)$ in the strong field regime, Eq. (5) yields $w L_{B}^{1 D}=\sqrt{C_{2}} l_{m} l_{e}$, in contrast to the diffusive expression $w L_{B}=\sqrt{3} l_{m}^{2}$ (to compare both expressions, boundary scattering effects have been neglected). Therefore, in direct analogy, we suggest to modify the diffusive expression $w L_{S}=$ $\sqrt{12} L_{\Omega}^{2}$ to a ballistic expression $w L_{S}=\sqrt{12} L_{\Omega} l_{e}^{*}$. We have retained the $\sqrt{12}$ proportionality constant as in diffusive wires but applied an effective mean-free path $l_{e}^{*} \equiv l_{e}\left(D / D_{2 D}\right)$ to account for boundary scattering. Using an effective meanfree path is here supported by the experimentally observed linear dependence of $w L_{S}$ on $D$. Thus, the analogy between magnetic vector potentials and spin-orbit effects leads us to model the extracted $L_{S}$ in our ballistic wires with $w L_{S}=$ $\sqrt{12} L_{\Omega} l_{e}\left(D / D_{2 D}\right)$. This expression is in accordance with our observation that $L_{S} \propto D / w$. Using $L_{\Omega}=\hbar^{2} /\left(2 m^{*} \alpha\right)$ and applying the expression for $w L_{S}$ to the data shown in Figs. 5(a) and 5 (b), we find $\alpha \approx 5 \times 10^{-12} \mathrm{eVm}$ for wires from both heterostructures.

The dependence $L_{S} \propto D / w$ in narrow ballistic wires can also be qualitatively understood by considering the effect of scattering off wire boundaries on the spin decoherence rate under the DP mechanism. The spin decoherence rate under the DP mechanism is inversely proportional to the momentum scattering rate $1 / \tau_{e},{ }^{36}$ as also expressed above in $1 / \tau_{S}^{2 D}=$ $\Omega^{2} \tau_{e} / 2$. Scattering from wire boundaries increases the overall scattering rate in narrow wires above that in the parent $2 \mathrm{DES}$. Using Matthiessen's rule, we estimate the effective overall scattering rate in narrow wires as $1 / \tau^{*}=\tau_{e}^{-1}+\tau_{w}^{-1}$ where $\tau_{w}$ represents the time associated with scattering from the wire boundaries which we approximate by the average time it takes an electron to diffuse a distance $w\left(\tau_{w} \approx w^{2} / D\right)$. For very narrow ballistic wires where $w \ll l_{e}$, a good approximation for the InAs wires considered here, scattering off wire boundaries is much more frequent than scattering in the bulk 2DES and leads to $\tau_{w} \ll \tau_{e}$ and, thus, $\tau^{*} \approx \tau_{w}$. Under the DP mechanism, the spin decoherence rate in the narrow ballistic wires can then be estimated by $1 / \tau_{S}^{1 D}=\Omega^{2} \tau^{*} / 2 \approx \Omega^{2} \tau_{w} / 2$. One then finds $L_{S}=\sqrt{D \tau_{S}^{1 D}} \approx L_{\Omega} l_{e}\left(D / D_{2 D}\right) / w$. Within a numerical constant, this qualitative expression agrees with the expression above. The qualitative approach shows that boundary scattering increases the overall scattering rate in narrow wires and can lead to a suppression of spin decoherence by motional narrowing $\left(\Omega^{2} \tau_{w} / 2<\Omega^{2} \tau_{e} / 2\right)$; however, the reduced $D$ arising from diffusive boundary scattering tends to lower $L_{S}$.

The Rashba parameter $\alpha$ can be obtained, separately, from Shubnikov-de Haas oscillations in magnetoresistance measurements. ${ }^{40,41}$ Rashba SOI causes a spin splitting of the Fermi surface, which can lead to beat nodes in the Shubnikov-de Haas oscillations. The spin-splitting energy $\epsilon$ is expressed as ${ }^{41,42}$

$$
\epsilon=\sqrt{\left[\hbar \omega_{c}\left(1-g^{*} m^{*} / 2 m_{e}\right)\right]^{2}+\left(2 \alpha k_{F}\right)^{2}}-\hbar \omega_{c},
$$

where $\omega_{c}=e B / m^{*}$ is the cyclotron frequency, $g^{*}$ is the effective Landé $g$ factor. Under Rashba SOI, beat nodes in the Shubnikov-de Haas oscillations occur at values of $B$ where $\epsilon$ is an odd multiple of $\hbar \omega_{c} / 2$. Shubnikov-de Haas oscillations measured for the unpatterned 2DES in heterostructure B are depicted in Fig. 6. One beat node corresponding to $\epsilon=\hbar \omega_{c} / 2$ and occurring at $B=0.55 \mathrm{~T}$ is observed. Using $g^{*}=-15,{ }^{29}$ we calculate a Rashba parameter $\alpha \approx 5.3 \times 10^{-12} \mathrm{eV}$ m from Eq. (6), in good agreement with $\alpha$ extracted from the observed dependence of $L_{S}$ on $D$ and $w$ in the Q1D model. The quantitative agreement between $\alpha$ extracted by the two separate methods should not be overemphasized. As discussed previously, the Q1D model does not include the short-time cutoff which, if included, would lead to slightly larger values of $L_{S}$ and, thus, a slightly lower value for $\alpha$; nor does it include the dimensional crossover which is expected to result in smaller values of $L_{S}$ and, hence, larger values for $\alpha$. The comparison, however, strongly supports the hypothesis that $w L_{S} \propto L_{\Omega} l_{e}\left(D / D_{2 D}\right)$. For quantitative comparisons, more rigorous theoretical models for both the dependence of $L_{S}$ on $D / w$ and for antilocalization in ballistic Q1D wires with diffusive boundary scattering, valid over a range of $w$ and $B$, are needed.

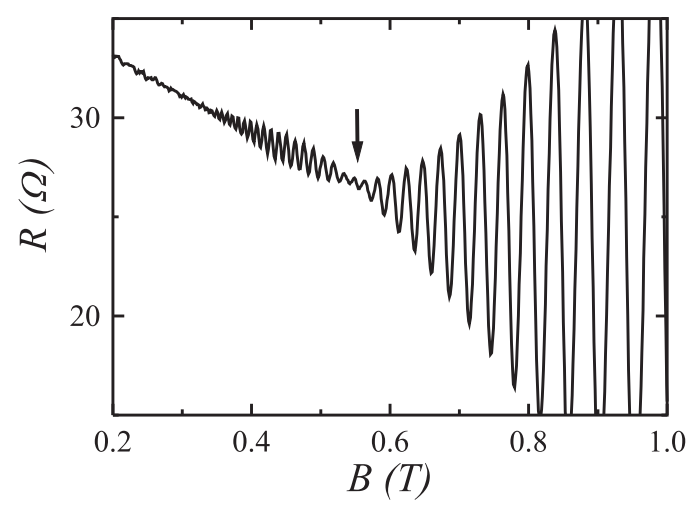

FIG. 6. Shubnikov-de Haas oscillations in the magnetoresistance of the unpatterned 2DES in heterostructure $\mathrm{B}(T=0.4 \mathrm{~K})$. The solid arrow highlights the observed beat node resulting from SOI in the 2DES. 
We note that for the narrowest wires from heterostructure B ( $w=0.17 \mu \mathrm{m}), L_{S}$ is smaller than predicted by the observed $L_{S} \propto D / w$ dependence for larger $w$. For extremely narrow wires, it is reasonable to expect that additional mechanisms for spin decoherence are active. For instance, due to the narrow band gap in InAs, the Elliott-Yafet spin decoherence mechanism ${ }^{36}$ may significantly affect $L_{S}$ in wires with very narrow $w$ where the overall electron scattering rate (intrinsic plus boundary) is high.

A detailed discussion of quantum phase coherence in the InAs wires is outside the scope of this paper. The linear dependence of $L_{\phi}$ on $D^{2 / 3} w^{1 / 3}$ in Figs. 5(c) and 5(d) indicates that the extracted $L_{\phi}$ are consistent with the Nyquist phase decoherence mechanism ${ }^{43,44}$ in Q1D wires. It is indeed predicted that for Nyquist decoherence in Q1D wires, $L_{\phi}^{3}=$ $\hbar^{2} v_{0} D^{2} w / k_{B} T$ (where $k_{B}$ denotes the Boltzmann constant). A deviation from the Nyquist prediction occurs at the widest $w$. Yet, such a deviation may be anticipated for wide $w$, as $L_{\phi}$ is expected to saturate to its $2 \mathrm{D}$ value as the wire approaches the $2 \mathrm{D}$ regime.

\section{CONCLUSIONS}

In narrow ballistic wires lithographically patterned on highmobility InAs 2DESs, the dependencies of the spin coherence length $L_{S}$ on wire width $w$ and diffusion constant $D$ are experimentally studied by analyzing the quantum corrections to the conductivity due to antilocalization. Using a Q1D antilocalization approach, the extracted values of $L_{S}$ are found to show an overall weak dependence on $w$, with $L_{S} \propto D / w$. Diffusive boundary scattering in the narrow InAs wires results in $D$ decreasing with decreasing $w$, affecting $L_{S}$ and the phase coherence length $L_{\phi}$. The often-observed increase in $L_{S}$ as $w$ narrows, arising from the $L_{S} \propto 1 / w$ dependence, is in the InAs wires largely canceled by the decreasing $D$.

\section{ACKNOWLEDGMENT}

The research is financially supported by the U.S. Department of Energy, Office of Basic Energy Sciences, Division of Materials Sciences and Engineering under Award No. DOE DE-FG02-08ER46532.
*Present address: Modern Microsystems, Inc., 13213 Locksley Ln., Silver Spring, MD 20904, USA; ray@ modernmicrosystems.com †heremans@vt.edu

${ }^{1}$ R. M. Lutchyn, J. D. Sau, and S. Das Sarma, Phys. Rev. Lett. 105, 077001 (2010).

${ }^{2}$ Y. Oreg, G. Refael, and F. von Oppen, Phys. Rev. Lett. 105, 177002 (2010).

${ }^{3}$ R. M. Lutchyn, T. D. Stanescu, and S. Das Sarma, Phys. Rev. Lett. 106, 127001 (2011).

${ }^{4}$ T. D. Stanescu, R. M. Lutchyn, and S. Das Sarma, Phys. Rev. B 84, 144522 (2011).

${ }^{5}$ S. Kettemann, Phys. Rev. Lett. 98, 176808 (2007).

${ }^{6}$ G. Bergmann, Phys. Rep. 107, 1 (1984).

${ }^{7}$ S. Hikami, A. I. Larkin, and Y. Nagaoka, Prog. Theor. Phys. 63, 707 (1980).

${ }^{8}$ S. V. Iordanskii, Yu. B. Lyanda-Geller, and G. E. Pikus, Pis'ma Zh. Eksp. Teor. Fiz. 60, 199 (1994) [JETP Lett. 60, 206 (1994)].

${ }^{9}$ W. Knap, C. Skierbiszewski, A. Zduniak, E. Litwin-Staszewska, D. Bertho, F. Kobbi, J. L. Robert, G. E. Pikus, F. G. Pikus, S. V. Iordanskii, V. Mosser, K. Zekentes, and Yu. B. Lyanda-Geller, Phys. Rev. B 53, 3912 (1996).

${ }^{10}$ M. M. Glazov and L. E. Golub, Semicond. Sci. Technol. 24, 064007 (2009).

${ }^{11}$ P. Santhanam, S. Wind, and D. E. Prober, Phys. Rev. B 35, 3188 (1987).

${ }^{12}$ A. E. Hansen, M. T. Björk, I. C. Fasth, C. Thelander, and L. Samuelson, Phys. Rev. B 71, 205328 (2005).

${ }^{13}$ Dong Liang, J. Du, and X. P. A. Gao, Phys. Rev. B 81, 153304 (2010).

${ }^{14}$ P. Roulleau, T. Choi, S. Riedi, T. Heinzel, I. Shorubalko, T. Ihn, and K. Ensslin, Phys. Rev. B 81, 155449 (2010).

${ }^{15}$ A. Wirthmann, Y. S. Gui, C. Zehnder, D. Heitmann, C. M. Hu, and S. Kettemann, Physica E (Amsterdam) 34, 493 (2006).

${ }^{16}$ A. A. Kiselev and K. W. Kim, Phys. Rev. B 61, 13115 (2000).
${ }^{17}$ T. P. Pareek and P. Bruno, Phys. Rev. B 65, 241305(R) (2002).

${ }^{18}$ A. W. Holleitner, V. Sih, R. C. Myers, A. C. Gossard, and D. D. Awschalom, Phys. Rev. Lett. 97, 036805 (2006).

${ }^{19}$ Th. Schäpers, V. A. Guzenko, M. G. Pala, U. Zülicke, M. Governale, J. Knobbe, and H. Hardtdegen, Phys. Rev. B 74, 081301(R) (2006).

${ }^{20}$ Y. Kunihashi, M. Kohda, and J. Nitta, Phys. Rev. Lett. 102, 226601 (2009).

${ }^{21}$ P. Lehnen, Th. Schäpers, N. Kaluza, N. Thillosen, and H. Hardtdegen, Phys. Rev. B 76, 205307 (2007).

${ }^{22}$ R. L. Kallaher, J. J. Heremans, N. Goel, S. J. Chung, and M. B. Santos, Phys. Rev. B 81, 035335 (2010).

${ }^{23}$ M. Rudolph and J. J. Heremans, Phys. Rev. B 83, 205410 (2011).

${ }^{24}$ D. C. Tsui, Phys. Rev. B 12, 5739 (1975).

${ }^{25}$ T. Mochizuki, R. Masutomi, and T. Okamoto, Phys. Rev. Lett. 101, 267204 (2008)

${ }^{26}$ M. Noguchi, K. Hirakawa, and T. Ikoma, Phys. Rev. Lett. 66, 2243 (1991).

${ }^{27}$ S. Bhargava, H. R. Blank, V. Narayanamurti, and H. Kroemer, Appl. Phys. Lett. 70, 759 (1997).

${ }^{28}$ G. Mihajlović, J. E. Pearson, S. D. Bader, and A. Hoffmann, Phys. Rev. Lett. 104, 237202 (2010).

${ }^{29}$ Landolt-Bornstein: Numerical Data and Functional Relationships in Science and Technology, edited by O. Madelung, M. Schulz, and H. Weiss (Springer, Berlin, 1984), Vol. 17a, pp. 297-310.

${ }^{30}$ C. W. J. Beenakker and H. van Houten, Phys. Rev. B 38, 3232 (1988).

${ }^{31}$ R. G. Wheeler, K. K. Choi, A. Goel, R. Wisnieff, and D. E. Prober, Phys. Rev. Lett. 49, 1674 (1982).

${ }^{32}$ K. K. Choi, D. C. Tsui, and K. Alavi, Phys. Rev. B 36, 7751 (1987).

${ }^{33}$ Ç. Kurdak, A. M. Chang, A. Chin, and T. Y. Chang, Phys. Rev. B 46, 6846 (1992).

${ }^{34}$ J. C. Licini, G. J. Dolan, and D. J. Bishop, Phys. Rev. Lett. 54, 1585 (1985). 
${ }^{35}$ A. Zduniak, M. I. Dyakonov, and W. Knap, Phys. Rev. B 56, 1996 (1997).

${ }^{36}$ I. Zutić, J. Fabian, and S. Das Sarma, Rev. Mod. Phys. 76, 323 (2004).

${ }^{37}$ M. Ferrier, A. C. H. Rowe, S. Guéron, H. Bouchiat, C. Texier, and G. Montambaux, Phys. Rev. Lett. 100, 146802 (2008).

${ }^{38}$ Y. A. Bychkov and E. I. Rashba, J. Phys. C: Solid State Phys. 17, 6309 (1984).

${ }^{39}$ T. Koga, J. Nitta, T. Akazaki, and H. Takayanagi, Phys. Rev. Lett. 89, 046801 (2002).
${ }^{40}$ A. M. Gilbertson, W. R. Branford, M. Fearn, L. Buckle, P. D. Buckle, T. Ashley, and L. F. Cohen, Phys. Rev. B 79, 235333 (2009).

${ }^{41}$ S. Faniel, T. Matsuura, S. Mineshige, Y. Sekine, and T. Koga, Phys. Rev. B 83, 115309 (2011).

${ }^{42}$ B. Das, S. Datta, and R. Reifenberger, Phys. Rev. B 41, 8278 (1990).

${ }^{43}$ B. L. Al'tshuler, A. G. Aronov, and A. Y. Zyuzin, Zh. Eksp. Teor. Fiz. 86, 709 (1984) [Sov. Phys.-JETP 59, 415 (1984)].

${ }^{44}$ B. L. Altshuler, A. G. Aronov, and D. E. Khmelnitsky, J. Phys. C: Solid State Phys. 15, 7367 (1982). 